\title{
Corrupción académica y su influencia en la democracia
}

\author{
MARTHA SAÑUDO* \\ Tecnológico de Monterrey (México) \\ msanudo@tec.mx \\ BONNIE J. PALIFKA ** \\ Tecnológico de Monterrey (México) \\ bonnie@tec.mx
}

\begin{abstract}
Resumen
Los estudiantes universitarios pueden contribuir a la calidad de la democracia de su país en la medida en que aprendan a distinguir cómo la corrupción se instala en su práctica cotidiana, la combatan y se comprometan a estar atentos a la honestidad de sus acciones y la veracidad de su discurso. Describimos las implicaciones de comprender a la democracia como gobierno por discusión y pasamos a proponer a la razón pública como el sine qua non de una democracia que busca hacer justicia social y que requiere ciudadanos dispuestos a debatir ideas con honestidad y rigor crítico, con debates públicos y pasión por la verdad. Estas ideas las contrastamos con los hallazgos de una encuesta sobre corrupción aplicada anualmente a estudiantes universitarios.
\end{abstract}

Palabras clave: Corrupción, deshonestidad académica, democracia, integridad.

\section{Academic corruption and the quality of democracy}

\begin{abstract}
University students may contribute to the quality of democracy in their country to the extent that they learn to distinguish how corruption is part of their day-to-day practices, they combat it and commit themselves to be attentive regarding the honesty of their actions and the veracity of their discourse. We describe the implications of understanding democracy as government by discussion; we then propose public reason as the sine qua non of a democracy that seeks social justice and requires citizens willing to debate ideas honestly, rigorously, and critically, in public debates and with a passion for the truth. We contrast these ideas with the findings from a yearly survey of university students.
\end{abstract}

Key words: Corruption, academic dishonesty, democracy, integrity.

\footnotetext{
* Doctora en Filosofía por la Universidad de Lovaina en Bélgica. Profesora Asociada del Departamento de Filosofía y Ética en el Tecnológico de Monterrey. Autora de "Business Ethics in Mexico: The Seeds of Justice" (2017) y "Corruption: history and future in the spotlight" (2016).

** Doctora en Economía por la Universidad de Texas en Austin. Profesora Asociada del Departamento de Economía en el Tecnológico de Monterrey y miembro del Sistema Nacional de Investigadores (nivel I). Co-autora de Corruption and Government: Causes, Consequences, and Reform, 2nd edition (2016) y ganadora del Premio Rómulo Garza 2017.
} 
1. LA DESHONESTIDAD ACADÉMICA ES EL INICIO DE LA FUTURA DESVINCULACIÓN MORAL

"El infierno son los otros"; ésta es una de esas frases atinadas de gente ilustre que permite a cualquier ciudadano parecer elocuente sobre el tema de los problemas humanos. La idea detrás de la frase es que los problemas de uno tienen su origen en los otros: son los otros los causantes de mi malestar; son los otros los que deben parar sus malas prácticas; son a los otros a los que se debe investigar y castigar; y en esa medida se podrá restituir el orden de nuestro mundo, en donde uno, es decir, el yo que juzga, no tiene cuentas pendientes ni culpa alguna. En este mismo estilo de excusarse uno mientras culpa al otro, es como frecuentemente se escucha hablar de la corrupción. Los corruptos son los otros. Los corruptos son los gobernantes, los políticos, los poderosos, los que han hecho que nuestros problemas sociales no puedan resolverse desde la iniciativa propia, pues el origen de estos problemas no nos incumbe, sino sus consecuencias, y por lo tanto nos levantamos como jueces de actos que son siempre iniciados por terceros. Efectivamente, jes indignante la corrupción de los otros! Mientras tanto, en este esfuerzo de juicio noble y persecutorio, se pueden mantener las acciones u omisiones propias, exentas de cualquier análisis que indique la posibilidad de que la corrupción se halle inserta al interior del propio mundo.

En la obra de teatro de Jean Paul Sartre, Huis Clos, de donde proviene la frase "el infierno son los otros", se dramatiza esta postura psicológica que permite neutralizar los juicios (la mirada) del otro, a través de enfatizar los tropiezos ajenos. Esta idea de "culpar a los otros por las penas comunes" es como un viraje a esa otra idea genial sartreana de "mala fe", en la que se describe al individuo que evade su responsabilidad arguyendo que otros le han quitado la libertad de actuar, y por tanto le parece imposible enfrentar la problemática de su propia existencia. Con el tema de la corrupción estos mismos mecanismos psicológicos que Sartre plantea en varias de sus obras, permiten a jóvenes señalar con su dedo índice inquisitorio a la llaga del otro, el otro que es corrupto, sin hurgar en sus propias prácticas cotidianas, en particular en lo que respecta a la copia y el plagio en la escuela y la universidad. Y estas prácticas ilegítimas efectuadas cotidianamente desde la niñez, son testimonio de fallas individuales y sociales repetidas que afectan a toda comunidad más allá del plano educativo. Como veremos más adelante, algunos estudiantes universitarios con quienes realizamos nuestra investigación, se sorprenden al advertir que muchos ciudadanos que ellos encuestaron no perciben incongruencia entre criticar la corrupción de la sociedad y admitir unos minutos más tarde ha- 
ber incurrido en sobornos; paradójicamente, muchos estudiantes no parecen notar incongruencia entre sorprenderse de la incongruencia de los ciudadanos corruptos y unos minutos más tarde reportar sus propias prácticas de copia, plagio y otros tipos de deshonestidad académica.

Es un lugar común afirmar que la escuela es el espacio socializador por excelencia, tanto como la familia, dado que en la mayoría de los países democráticos los niños pasan durante su infancia tanto tiempo en la escuela como con sus familias. Desde Piaget hasta los numerosos y diversos trabajos contemporáneos sobre educación y desarrollo moral, se observa que, en términos de la formación social del niño, la escuela constituye su primer encuentro con la forma en que la sociedad se organiza. Las prácticas escolares enseñan al niño la forma en que los individuos interactúan, se jerarquizan distintos actores, se despliega la autoridad, y cómo se relacionan distintas instituciones como son la escuela, la familia, el gobierno y las iglesias. Por tanto, es en la escuela donde el niño aprende que la arena pública es el lugar donde se promueve el bien social, desarrollando valores cívicos y culturales, o bien, se aprende que lo público es una plataforma donde se establecen competencias injustas y falseadas, rebatingas y golpes bajos, en un ambiente de impunidad.

En la investigación que realizamos para este trabajo, encuestamos a alumnos sobre si la copia en exámenes era común en cada uno de los niveles escolares cursados. En la gráfica siguiente se observa que la deshonestidad académica se hace más común mientras avanza el alumno, es decir, se reporta menos deshonestidad académica en la primaria que en la secundaria, y menos en la secundaria que en la preparatoria. Esto implica que la deshonestidad, si no se reprime temprano, se convierte en alud. En efecto, la deshonestidad académica puede ser la primera "pendiente resbaladiza" (Welsh, Ordóñez, Snyder \& Christian, 2015) que enfrentan los jóvenes.

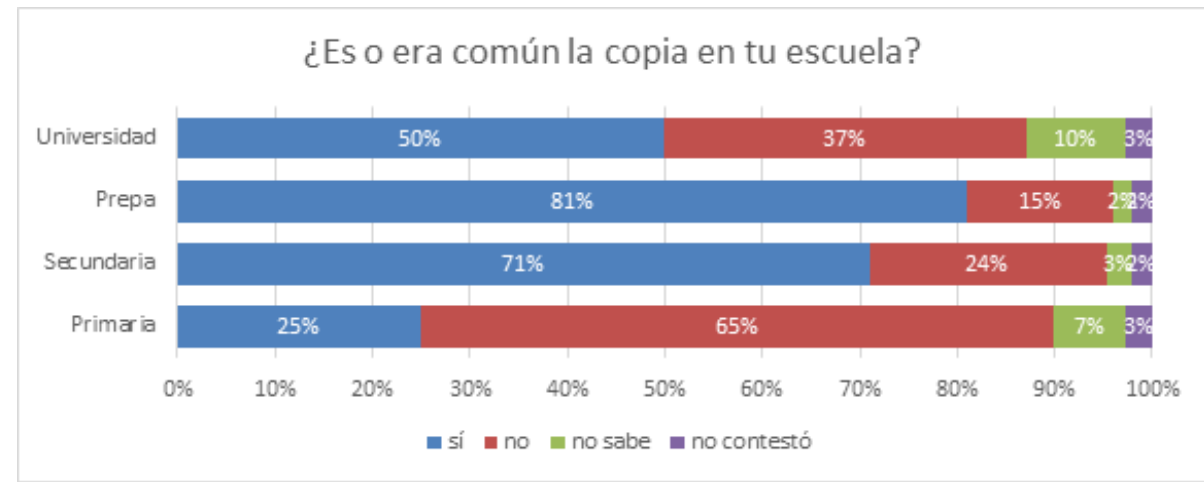

Fuente: elaborada por las autoras como resultado de las entrevistas reportadas abajo. 
Aunque en la gráfica parece ser menos común la copia en la universidad que en la preparatoria, se repite el mismo patrón dentro de la universidad: conforme el alumno avanza en semestres cursados, más percibe que la copia sea común. Por ejemplo, de los alumnos de noveno semestre, ya cerca de terminar sus estudios, el $83 \%$ indica que es común la copia. Este resultado es parecido al observado por Denisova-Schmidt, Huber \& Leontyeva (2016) para alumnos universitarios en Rusia, quienes al avanzar en sus estudios se volvían más tolerantes de una variedad de prácticas deshonestas. Los autores argumentan que los estudiantes se vuelven más "pragmáticos" (Denisova-Schmidt et al., 2016: 9). En otras palabras, los alumnos aprenden a dejar a un lado su juicio moral al exponerse a una realidad en que la falta de integridad, lejos de ser castigada, les da una ventaja.

Como la pregunta anterior representa una percepción del alumno, debemos comprender que dicha percepción puede ser influenciada por varios factores, por lo que decidimos preguntar también si ellos mismos habían copiado en exámenes, durante cada nivel escolar, para notar la consistencia del posible patrón. Como se observa en la siguiente gráfica, sí se repite el patrón: entre más avanzan en el sistema educativo, más recurren a la copia, con una baja a nivel universitario. Sin embargo, esta reducción a nivel universitario puede deberse a que a la hora de hacer estas encuestas sus estudios de preparatoria ya están terminados y sus estudios universitarios están todavía en curso y no les es posible evaluar de golpe todos sus estudios universitarios. Esta intuición nos llevó a desagregar las encuestas por semestre y vimos que efectivamente, si tomamos en cuenta las encuestas solamente de los alumnos en semestres avanzados, de séptimo en adelante, aparece el mismo porcentaje de deshonestidad en la universidad que en la prepa. Entre los de noveno, las encuestas arrojan que todos $(100 \%)$ han copiado al menos una vez y $67 \%$ han copiado varias veces.

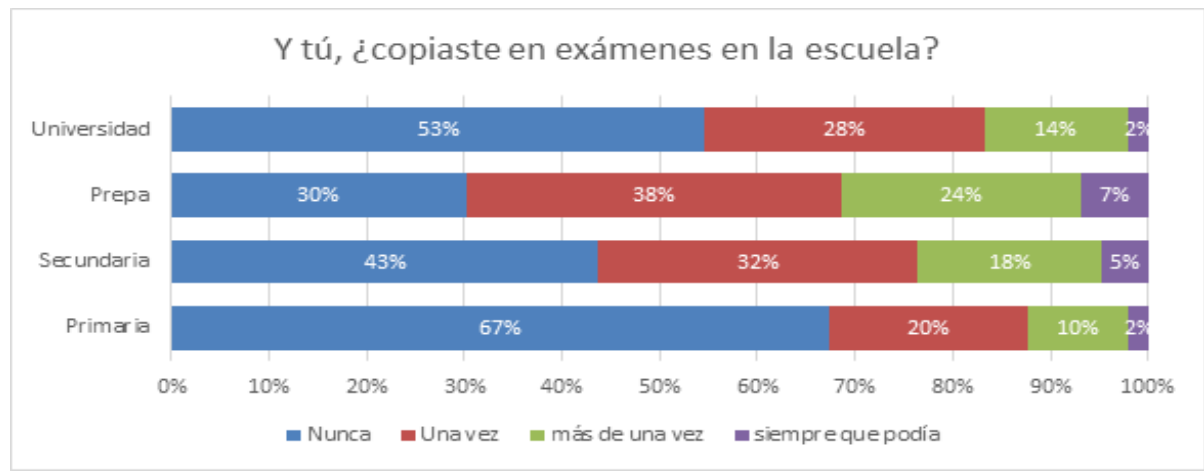

Fuente: Autoras. 
Nota: en cada categoría, un alumno marcó "no sé" o "no contestó", representando un $0.68 \%$ de la muestra. Se omitieron estas categorías de la gráfica por cuestiones de claridad. Por lo tanto, las categorías aquí mostradas no suman al 100\%.

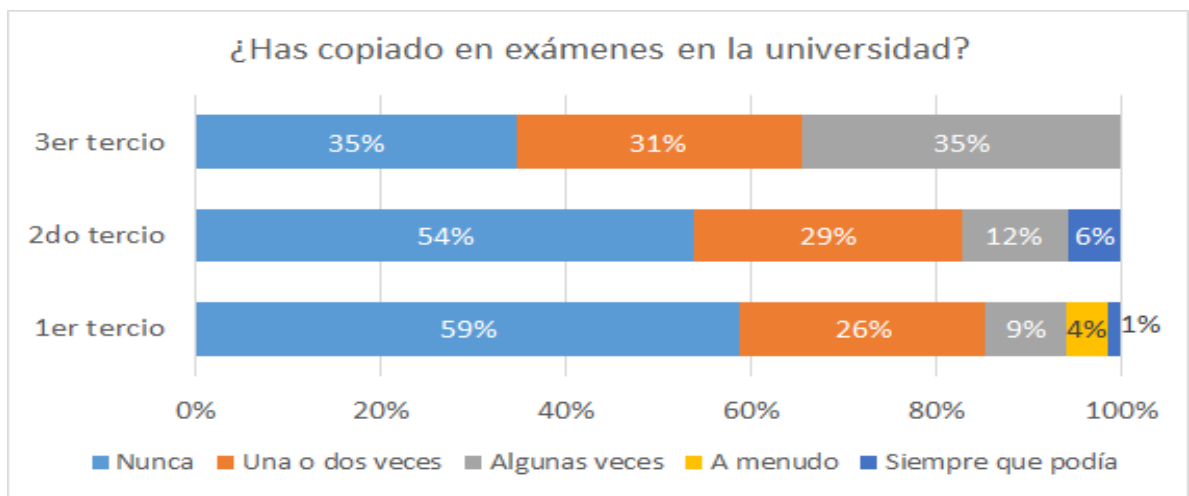

Fuente: Autoras.

Asimismo, en las siguientes gráficas comparamos las respuestas de los estudiantes que no perciben las trampas como comunes entre sus compañeros contra las de los alumnos que sí perciben la trampa como común. Observamos que los estudiantes que perciben la trampa como común son mucho más propensos a participar en actos de deshonestidad académica: la proporción que ha hecho trampa una o dos veces aumenta de $22 \%$ al $35 \%$; los que reportan haber hecho trampa "varias veces" sube de $2 \%$ a $25 \%$; "muchas veces" incrementa de $0 \%$ a 3\% y "siempre que puedo" se mantiene en $3 \%$.

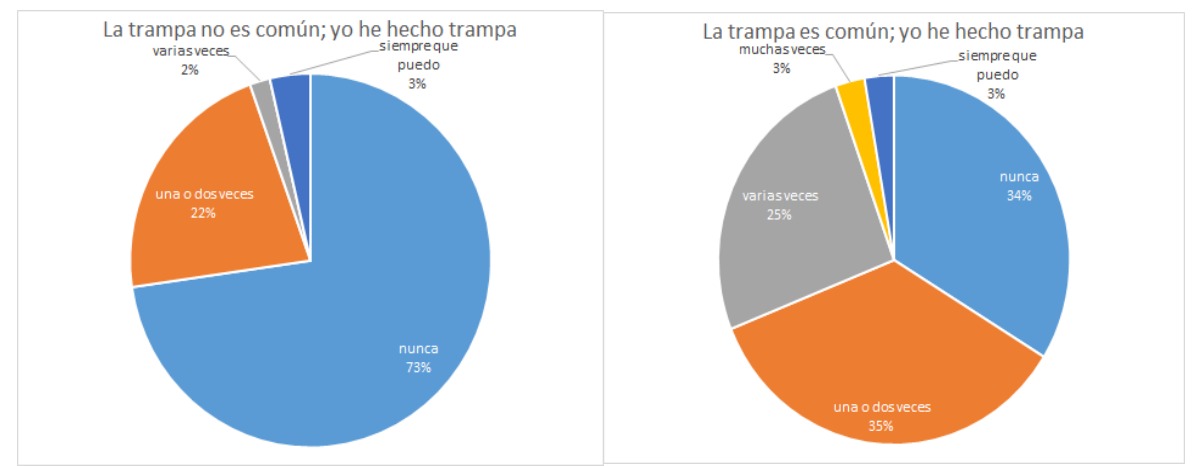

Fuente: Autoras.

Es por este marco de referencia, de la escuela como socializadora del niño, que se estudian las prácticas de deshonestidad académica como una génesis de la desvinculación moral que ocurre entre las prácticas corruptas y una visión moral que permita controlar la socialización negativa. Es decir, al ignorar la deshonestidad académica como una práctica contraria a 
su formación moral, los alumnos aprenden a desvincular ciertas prácticas propias de su juicio moral y esto genera una sociedad corrupta y por tanto una democracia ineficiente, como veremos más adelante. Por deshonestidad académica entendemos

cualquier comportamiento intencional que busque un beneficio académico, económico, afectivo o de cualquier otra índole que vaya en contra de los principios éticos de las instituciones educativas y, en consecuencia, de la sociedad; es un fenómeno que altera y afecta el aprendizaje y desempeño académico, el proceso de evaluación, las relaciones entre profesor-alumno y alumno-alumno, así como la integridad de la institución y, por lo tanto, la formación en valores personales y grupales (Diez-Martínez, 2015: 3).

Dentro de las instituciones educativas, la deshonestidad académica tiene, como todas las acciones corruptas, la cualidad de poder corromper -es decir, roer, dañar o pervertir- la misión para la cual se instituyó dicha institución. En otras palabras, con la deshonestidad académica se corrompe la institución de la educación pues socava la posibilidad de evaluar con objetividad el resultado de la práctica educativa. Si los alumnos de un curso obtienen calificaciones aprobatorias, pero no se sabe si estos alumnos obtuvieron o no obtuvieron los conocimientos y habilidades propias del curso, difícilmente podemos organizar los cursos siguientes, o en su caso, garantizar cierto estándar de conocimiento a los futuros empleadores de dichos alumnos.

Por otro lado, la deshonestidad académica, como una práctica cotidiana, corroe la formación ética del alumno en el sentido de inhibir el juicio crítico de los alumnos, y adoptar una desvinculación entre lo que el alumno comprende que es la conducta deseable y su práctica real. El artículo seminal de Albert Bandura (1999), "Moral disengagement in the perpetration of inhumanities" (La desvinculación moral en la perpetuación de inhumanidades) muestra los sesgos cognitivos que permiten a personas ordinarias cometer atrocidades humanas, por ejemplo, desvinculando las acciones crueles del control moral a través de mecanismos psicológicos como pueden ser el utilizar un lenguaje de eufemismos ${ }^{1}$ o expresiones que deshumanizan a las víctimas. El alcance del trabajo de Bandura radica en mostrarnos los mecanismos psico-sociales que se ponen en juego para que una acción que ordinariamente sería moralmente condenada, pueda verse moralmente

$1 \quad$ Como bien argumenta Johnston (2014: 63): "The language of corruption... is rich in euphemisms and evasions...that reflect and encourage continued cynicism...." Mialon \& Mialon (2013) desarrollan un modelo de teoría de juegos para demostrar que la indirecta puede ser un equilibrio. Presentan la corrupción como un ejemplo y la ironía como otro. 
justificada a los ojos de los perpetradores de dicha acción. Llevando estos hallazgos al tema que nos concierne, podemos advertir que los controles ordinarios para rechazar y condenar la deshonestidad académica, son fácilmente desplazados cuando se desestima el impacto individual y las consecuencias sociales de esta práctica. En específico presentaremos cómo la deshonestidad académica afecta la calidad de la democracia que se busca promover en una ciudadanía, al inhibir el juicio crítico y obstaculizar la búsqueda por la imparcialidad y la verdad.

\section{LA DESHONESTIDAD ACADÉMICA Y LA DEMOCRACIA}

En La idea de justicia, Amartya Sen (2012) promueve la definición de la democracia como razonamiento público y gobierno por discusión, una definición que Sen toma prestada de John Stuart Mill. En el segundo capítulo de Sobre la libertad, Mill (1999 -original en inglés, 1859) propone las ventajas de poner a discusión cualquier idea, por más absurda o hereje que parezca, estableciendo de esta forma a la libertad de palabra o de discusión (freedom of speech) como una de las características fundamentales del pensamiento liberal vigente hasta nuestros días. Al permitir la discusión y rechazar la censura, se permite distinguir distintos puntos de vista y descubrir divergencias de intereses. Sobre todo, es a través de las discusiones donde caemos en cuenta que nuestra opinión propia no es infalible. Mill lo argumenta así:

No dejar conocer una opinión, porque se está seguro de su falsedad, es como afirmar que la propia certeza es la certeza absoluta. Siempre que se ahoga una discusión se afirma, por lo mismo, la propia infalibilidad [...] En efecto, mientras cada cual sabe muy bien que es falible, solamente un pequeño número de individuos juzgan necesario tomar precauciones contra la propia falibilidad, o bien admitir la hipótesis de que una opinión de la cual se sienten seguros puede ser uno de los casos de error a que se reconocen sujetos (Mill, 1999: 77).

Para Mill es por tanto claro que un gobierno democrático debe asegurar la libertad de discusión. Sen, por su parte, presenta esta práctica de la libertad de discusión más bien como el sine qua non de la buena democracia, proponiendo que la discusión pública y la posibilidad de poner ideas al escrutinio público, es la única forma de garantizar cierta objetividad a los juicios morales, que a su vez garantizan la legitimidad de las políticas elegidas democráticamente. Es decir, la objetividad de los juicios morales, una objetividad que es de grado y no absoluta, es clave para la buena democracia porque la objetividad de los juicios permite la imparcialidad en 
la justicia y toda buena democracia busca la justicia. Así, Sen propone que, para buscar la justicia con eficacia, tenemos que desarrollar

la capacidad de ser razonables al mantener la mente abierta a la información, al reflexionar sobre los argumentos de diferentes procedencias, y al participar en deliberaciones y debates de carácter interactivo sobre las cuestiones fundamentales [de una sociedad] (Sen, 2012: 73).

Esta libertad de expresión y oportunidades para el debate público contrastan con el ambiente que se promueve en instituciones educativas donde se condona la copia y la deshonestidad académica, pues en ellas se promueve la complicidad y la confabulación, más que el pensamiento crítico y el interés por la expresión y escrutinio libre de ideas. En un ambiente donde abundan la copia y el plagio, es la mentira lo que impera y no la objetividad ni la búsqueda de la imparcialidad o la justicia. Por ende, las capacidades que Sen apunta en la cita de arriba, difícilmente se despliegan en su total potencialidad en nuestros alumnos. Y como consecuencia, la calidad de la democracia también se verá inevitablemente afectada, pues los ciudadanos no serán capaces de buscar la justicia con imparcialidad a menos de que tomen conciencia de la necesidad de comprometerse a la honestidad de sus acciones y a la veracidad de su discurso. Este concepto de una democracia basada en la participación honesta de sus ciudadanos, especialmente en asuntos de anticorrupción, remonta al menos hasta la Atenas antigua (Taylor, 2018). Sen afirma que una "justicia sin discusión" puede ser difícilmente una justicia, en el sentido de que una verdadera democracia debe dar cabida a oportunidades para la discusión, pues es a través del discurso público que los valores sociales se legitiman como normas y prioridades a seguir (Sen, 2012: 366-367).

Por todo lo anterior, proponemos que los estudiantes universitarios serán capaces de contribuir a la calidad de la democracia de nuestro país en la medida en que aprendan a distinguir cómo la corrupción se ha instalado en su visión moral desde la niñez y deseen combatirla. La relación entre la corrupción y la democracia ha sido explorada de varias formas. Por ejemplo, Canache \& Allison (2005) ponen en evidencia que la corrupción no disminuye el apoyo de los latinoamericanos por la democracia, aunque sí por la administración gubernamental que perciben como corrupta. Por otro lado, invirtiendo la causa y el efecto, el desarrollo de la democracia puede ser una fuerza en contra de la corrupción. Por ejemplo, la democracia puede mitigar los efectos negativos de la corrupción (Drury, Krieckhaus \& Lusztig, 2006). Otros estudios encuentran que el nivel de desarrollo democrático reduce la persistencia del crimen organizado (Bus- 
caglia \& van Dijk, 2003) y el nivel de corrupción (Treisman, 2000; Lederman, Loayza \& Soares, 2006). Otros argumentan que no es la democracia por sí misma, sino el proceso de democratización (Johnston, 2012) o el conjunto de políticas apropiadas (Glaeser, La Porta, Lopez-de-Silanes \& Shleifer, 2004), lo que reduce los niveles de corrupción. Así, podemos concluir que la relación entre la corrupción y la democracia es compleja, pero en general la presencia de una disminuye la otra.

Una parte intrínseca del desarrollo democrático, entonces, independientemente de que si sea causa o efecto, es la necesidad de detectar y eliminar, o reducir, las prácticas de corrupción. Aunque hay muchas formas de combatir la corrupción (véase Rose-Ackerman \& Palifka, 2016), una parte importante es un cambio cultural: cambiar la mentalidad de la población, para que sea menos tolerante de las prácticas corruptas y deshonestas. En este sentido, Basu (2011) argumenta que la clave es promover la moralidad y la honestidad para combatir la corrupción. Históricamente, éste ha sido una parte importante de algunos movimientos anticorrupción: por ejemplo, a través de manuales para gobernantes en Arabia en los siglos X y XI (van Berkel, 2018) y espejos con mensajes morales para príncipes en Inglaterra en los siglos XIII-XVI (Watts, 2018).

En aras de investigar las formas como se instala la corrupción en la práctica cotidiana estudiantil, realizamos con estudiantes universitarios la actividad que a continuación detallamos.

3. ACTIVIDADES REALIZADAS CON ESTUDIANTES PARA DETERMINAR LA RELACIÓN ENTRE LA CORRUPCIÓN Y LA DESHONESTIDAD ACADÉMICA

Durante cuatro años (2015, 2016, 2017 y 2018) hemos llevado a cabo un taller de una semana sobre la corrupción en Monterrey, México. La cantidad de alumnos participantes y la programación de actividades específicas han variado cada año, pero con una misma organización. La semana comienza con cátedras sobre la corrupción: definiciones, formas de medirla, causas, consecuencias y estrategias para su combate. Durante el primer día del taller, los alumnos contestan una encuesta. Durante varios días, los participantes aplican la encuesta a otros alumnos y al público en general. Aunque el enfoque de la encuesta es la corrupción, se incluyen preguntas sobre la deshonestidad académica, con el propósito de establecer si existe una relación entre la tolerancia hacia la deshonestidad académica, por un lado, y las actitudes hacia la corrupción y el pago de sobornos, del otro. Al final de la semana, los alumnos entregan tanto las respuestas de esas encuestas como una reflexión sobre lo aprendido y vivido durante el taller. En el 2018, adicionalmente entregaron videos producidos por equipos, como detallamos más adelante. 
En sus reflexiones, los alumnos ponen en evidencia que, efectivamente, solemos juzgar con severidad las acciones negativas de los demás, a pesar de que nosotros también realizamos similares acciones negativas. Por ejemplo, una alumna escribió:

... aun cuando criticamos ciertas acciones de los demás, seguimos actuando de esa misma forma.... [D]e 37 personas a las que se les hizo la pregunta ¿Consideras que copiar de otro alumno durante un examen es trampa? 21 de ellas contestaron que era trampa grave, 12 encuestados lo consideran trampa moderada, mientras que 4 piensan que sí es trampa aunque ligera; con estos datos se puede decir que el $100 \%$ de los entrevistados consideran que es trampa copiar a otro alumno durante un examen. No obstante, al hacer la pregunta chas copiado en exámenes en la primaria, secundaria, preparatoria o universidad? Solo 8 encuestados dijeron no haber copiado nunca en ningún nivel escolar mientras que uno dijo no saberlo; es decir, el $75 \%$ de las personas que respondieron la encuesta ha copiado en algún examen aun cuando lo considera como trampa...

Otro alumno observa que la distancia entre el gobierno y el pueblo permite que consideremos corrupto al gobierno, sin reflexionar que nosotros también lo somos:

Lo que he aprendido acerca de nuestra sociedad mexicana es que el $99.9 \%$ de las personas encuestadas odian la corrupción, pero ellos no hacen nada para combatirla, solo piensan en que el gobierno es corrupto y critican al gobierno, pero lo que ellos no ven es que el gobierno es corrupto porque nosotros los ciudadanos somos corruptos y esto hace que la corrupción sea parte de la cultura mexicana. Por lo que para cambiar nuestra sociedad y lograr que disminuya la corrupción de los mexicanos, debemos de empezar por uno mismo; y la corrupción va desde la evasión de impuestos hasta los actos de deshonestidad académica.

Este mismo pensamiento - que el gobierno es corrupto porque nosotros también lo somos- se repite en la reflexión de otro alumno:

... resulta irónico el hecho de que como ciudadanos manifestamos nuestra inconformidad con la situación actual de nuestro país, reconociendo la injerencia que la corrupción puede tener en dicha situación, pero al mismo tiempo somos los mismos que, con nuestros actos, alimentamos al monstruo del que nos quejamos.

Somos aquellos que señalamos la deshonestidad de nuestros funcionarios públicos con la misma mano con la que le damos la mordida al tránsito. Aquellos que, enrabiados e indignados, observamos el compadrazgo operante que nos victimiza en algún trámite burocrático, con los mismos ojos 
con los que tratamos de mirar las respuestas del examen de nuestro compañero de al lado. Así pues, es sorprendente saber que quizá nuestro problema principal no es la falta de conciencia, sino la falta de congruencia.

Otro tema evidente es la relación entre las prácticas deshonestas en la escuela y los actos corruptos en la sociedad, remarcando la incongruencia entre lo dicho y lo hecho:

... todos se quejan de los servidores públicos, policía e incluso hasta de la iglesia, sin embargo, al momento de preguntarles si ellos mismos en algún momento habían recurrido a algún tipo de copia en sus asuntos escolares, la mayoría contestó positivo, lo cual significa que la manera de pensar de la mayoría de los mexicanos no va de acuerdo a sus acciones, y esto mismo es lo que sucede en los altos mandos del país.... Con esta semana, aprendí que las personas no se respetan ni a ellos mismos ya que dicen creer y sostener una cosa, pero en sus acciones reflejan otra.

Este concepto se repite en otra reflexión:

a pesar de que reprueban estas conductas, la mayoría de las personas entrevistadas participó en al menos un acto corrupto o tolera ciertas prácticas corruptas, [...] considero que la sociedad [...] debe cambiar su mentalidad y volverse un poco más congruente con sus pensamientos y sus acciones.

Una constante en los comentarios hechos por los alumnos, es la autoreflexión y toma de conciencia respecto a descubrir que la democracia opera en función de los ciudadanos; es decir, no hay buena democracia a menos de que haya buenos ciudadanos demócratas. Como observa una alumna:

...mucha gente culpa al gobierno o al país en general por [el] alto porcentaje de corrupción que tiene México, pero no se dan cuenta que los mismos que causan esto son los propios ciudadanos y que no podemos culpar a los gobernantes por algo que nosotros mismos hacemos.

Otro alumno hace hincapié en el papel del ciudadano en la corrupción y la anticorrupción:

Pienso que muchas veces uno se pregunta ¿y qué hacer para combatir la corrupción? y muchas veces nos sentimos atrapados y sin respuesta; pero esta semana en verdad me ayudó a ver que la corrupción está en todas partes, y por lo tanto también depende-más de lo que pensamos-de nosotros atacarla desde las acciones tan simples y pequeñas como estacionarnos 
donde nos corresponde y no dar pagos extraoficiales. Así hacemos que la bola de nieve buena crezca y no la mala.

Otras estudiantes muestran una clara concientización, aseverando una "que a lo largo de la semana [...] dejé de ver normales acciones que no son buenas como por ejemplo el descargar música sin pagar o leer resúmenes en vez de lecturas originales, ya que es algo que no veía mal pero ahora entendí que no son buenas prácticas" y otra que "[e]n mi opinión se puede hacer un cambio, solo es cuestión de día a día hacer lo que nos corresponde basándonos por nuestros valores y ética".

Estas reflexiones demuestran que existe una desvinculación entre el pensar y el hacer cotidiano de la ciudadanía mexicana, y además demuestran que la inconsciencia colectiva puede fácilmente convertirse en un acto de conciencia a través de una reflexión guiada. Es decir, los alumnos que tomaron el taller, en tan solo cuatro días lograron un verdadero despertar: reconocieron que cada quien contribuye a una sociedad corrupta o deshonesta con sus acciones corruptas o deshonestas y que esto a su vez impacta en la funcionalidad de una democracia. Es a través del despertar de su conciencia que ellos mismos pueden concluir que una forma de contribuir al fortalecimiento de una sociedad democrática sería rechazar la deshonestidad y la corrupción, y luego pasar a esperar que el gobierno elegido actúe para promover los intereses del bien común. Así pues, la educación juega un papel primordial en la formación de los ciudadanos y en el buen funcionamiento de la democracia.

La mejor forma de concretar un aprendizaje es enseñarlo a otros. En este sentido, para la última edición del taller, realizada en septiembre 2018, trabajamos con la campaña "Soy Honesto Hoy", una campaña pública que pretende atacar la corrupción a través de la participación cotidiana de los ciudadanos $^{2}$. Esta campaña celebró su lanzamiento el 20 de septiembre ${ }^{3}$; la actividad con los alumnos tomó lugar del 24 al 28 del mismo mes. En esta ocasión, además de las actividades descritas arriba, los estudiantes prepararon cortometrajes vinculados con la campaña. Las temáticas que abordaron en los vídeos demuestran la internalización de la importancia del actuar honesto para una democracia más plena y una sociedad menos corrupta.

En uno de los videos, los estudiantes abordan la frase común, "No pasa nada." Muestran una persona que cruza la calle cuando la señal indica

2 El lector puede encontrar más sobre esta campaña en https://www.soyhonestohoy.mx/.

3 Juan Carlos Rodríguez y Leonardo González, "Lanzan 'Soy Honesto Hoy", Reforma, 20 de septiembre de 2018, https://www.reforma.com/aplicaciones/articulo/default.aspx?id $=1495142 \& v=2$. 
no cruzar, una alumna que decide copiar en un examen y un constructor que consigue permisos a través del soborno. En cada caso, ante la disyuntiva, la persona involucrada piensa, "No pasa nada." Luego gritan, "¡Sí, pasa!" y muestran un caso periodístico de una persona atropellada, un maestro reprobando a la alumna y otra noticia sobre una construcción que se colapsó, causando muertes.

Un segundo video critica la violación de normas, terminando con las siguientes frases: "La corrupción nos lleva a la hipocresía. Piensa bien antes de actuar". Esta misma hipocresía es el motivo de otro video, en el que los alumnos señalan: "Cuando tú mientes, robas y optas por la vía fácil [,] no eres ingenioso, eres corrupto" ". Otro equipo termina su video con una invitación: "La decisión es tuya. Te invito a hablar con la verdad, a enfrentar a la corrupción. Te invito a Ser Honesto Hoy".

Estos estudiantes, a través de sus videos, demuestran su pensamiento crítico al destacar la incongruencia social entre la reprobación de actos de corrupción mientras se participa en ellos. Asimismo, evidencian la forma en que la integridad significa desarrollar congruencia entre el pensar y el hacer, rechazando la corrupción con las decisiones que tomamos.

\section{EVIDENCIA ESTADÍSTICA DE LA INCONGRUENCIA RESPECTO A LA DES- HONESTIDAD ACADÉMICA}

El patrón que observó una alumna en las encuestas que ella aplicó (citada arriba), se replica entre las que contestaron los mismos alumnos en el salón. Ninguno cree que copiar de otro alumno durante un examen no sea trampa, pero casi todos aceptan haberlo hecho al menos una vez en el transcurso de sus estudios. En la tabla siguiente tomada del 2015, observamos la distribución conjunta de dos preguntas: primera, ¿Consideras que sea trampa copiar de otro alumno durante un examen?; y segunda, ¿Con qué frecuencia has copiado de otro alumno durante un examen? En cada caso, tenían la opción de no contestar - pero nadie eligió esta respuesta para estas preguntas.

\footnotetext{
4 Video disponible en https://drive.google.com/file/d/1qTsLtRWT58u4pHA1acgcW2yUiSG1M51W/view

5 Video disponible en https://drive.google.com/file/d/1MadgtCqmINvpFrw2Bta0Lb5FfeaVhnxO/view?pli=1

Video disponible en https://www.youtube.com/watch?v=HIUjsj4DUK4\&feature=youtu.be
} 


\begin{tabular}{|c|c|c|c|c|c|c|}
\hline \multirow{8}{*}{$\begin{array}{l}\text { ¿Con } \\
\text { qué fre- } \\
\text { cuencia } \\
\text { has co- } \\
\text { piado de } \\
\text { otro } \\
\text { alumno } \\
\text { durante } \\
\text { un exa- } \\
\text { men? }\end{array}$} & & \multicolumn{5}{|c|}{$\begin{array}{l}¿ \text { Consideras que copiar de otro alumno durante un exa- } \\
\text { men sea trampa? }\end{array}$} \\
\hline & & $\begin{array}{l}\text { No es } \\
\text { trampa }\end{array}$ & $\begin{array}{l}\text { Tramp } \\
\text { a ligera }\end{array}$ & $\begin{array}{c}\text { Trampa } \\
\text { mode- } \\
\text { rada }\end{array}$ & $\begin{array}{l}\text { Tramp } \\
\text { a grave }\end{array}$ & $\begin{array}{c}\text { To- } \\
\text { tal }\end{array}$ \\
\hline & Nunca & 0 & 1 & 4 & 32 & 37 \\
\hline & Una vez & 0 & 2 & 13 & 27 & 42 \\
\hline & $\begin{array}{l}\text { Más de } \\
\text { una vez }\end{array}$ & 0 & 14 & 28 & 23 & 65 \\
\hline & $\begin{array}{l}\text { Muchas } \\
\text { veces }\end{array}$ & 0 & 0 & 2 & 1 & 3 \\
\hline & No sé & 0 & 0 & 1 & 0 & 1 \\
\hline & Total & 0 & 17 & 48 & 83 & 148 \\
\hline
\end{tabular}

Fuente: Autoras.

Todos aceptan que es trampa, aunque difieren en el grado de gravedad. Un 56\% (83 alumnos) aceptan que es una trampa grave; entre éstos están el 86\% (32 de 37 alumnos) de los que nunca han copiado de sus compañeros en un examen. Quizás su convicción de que es una trampa grave, es lo que les ha permitido resistir la tentación en la que caen otros. Por el contrario, de los que creen que es una trampa ligera, el 82\% (14 de 17 alumnos) han copiado en exámenes más de una vez. ¿Será que creen que "no pasa nada" y por ende copian, o que el hecho de haber copiado les hace ser más tolerantes ante ese comportamiento? Esta es una pregunta de causa y efecto, con un aspecto psicológico, que estos datos no nos permiten contestar. Del otro extremo, muy irónicamente, los tres alumnos que aceptan haber copiado en exámenes "muchas veces" consideran que la copia es una trampa moderada ( 2 alumnos) o grave ( 1 alumno). De hecho, hay 54 alumnos (36\% del total) que admiten haber copiado "más de una vez" o "muchas veces" a pesar de considerarlo una trampa de moderada a grave.

Realizamos estas mismas preguntas para un total de trece prácticas académicas, las mismas prácticas estudiadas por Balbuena y Lamela (2015) en Filipinas. Ninguna otra práctica presenta un contraste tan fuerte en nuestros datos, pero para todas se observa el mismo fenómeno: alumnos 
que clasifican la práctica de la copia como trampa y que a su vez aceptan haberla cometido.

Es interesante contemplar también algunas de las respuestas a la pregunta abierta incluida en la encuesta: ¿Qué opinas de los alumnos que hacen trampa para pasar las materias? Esta pregunta viene después de la serie de preguntas en que clasifican las prácticas como trampa, pero antes de la pregunta sobre sus propias prácticas. Mientras que muchos opinaron que era injusto para los alumnos que estudian, o que los mismos alumnos deshonestos algún día sufrirán las consecuencias, algunos alumnos encontraron una relación entre la deshonestidad académica y la situación del país:

- son flojos, irresponsables, inmaduros y un potencial peligro para el desarrollo del país

- hacen un peor daño a ellos y el país

- son un problema para la industria, para el país, para todo

- dañan al país

- dañan la integridad del país

- están pasando sin esfuerzo y contribuyen a la corrupción y no benefician a la sociedad

- afectan a la sociedad en sí

- personas sin valores que significan la pobre evolución de la sociedad

Dado que la encuesta llevada a cabo trata sobre actitudes y prácticas que son reprobables, es posible que los participantes se auto censuraran y no quisieran compartir honestamente sus pensamientos y experiencias (¡lo que en sí mismo constituye una paradoja al estilo de cualquiera de las famosas paradojas de Zenón!). Sin embargo, como era un taller sobre la corupción, los estudiantes estaban ya familiarizados con la alta incidencia de corrupción en México y el mundo, y podríamos intuir que por lo tanto estarían dispuestos a compartir su conocimiento particular al respecto, y la forma como ellos constituyen parte del problema y responder honestamente en la encuesta como en la discusión en el salón.

Otra consideración es la auto-selección. Debido a que era un taller sobre la corrupción, la mayoría de los participantes tenían cierto interés en el tema. Por lo tanto, no podemos aseverar que sus respuestas sean representativas ni siquiera de los alumnos en la misma universidad, mucho menos de la sociedad regiomontana o de la mexicana. Sería interesante realizar un estudio parecido con una muestra más representativa. 


\section{CONCLUSIONES}

Un requisito para modificar conscientemente una práctica cualquiera es reconocer la forma en que esta práctica se instala en las conciencias de los practicantes. Si los alumnos y las instituciones educativas tomaran conciencia de la pandemia de deshonestidad académica que nos asola, y del riesgo existente en desestimar la corrupción del carácter moral de los alumnos al condonarla, podríamos evitar una de las causas - comprobadas por este estudio- de la desvinculación moral entre lo que se cree y lo que se hace en nuestra sociedad.

Para mejorar la calidad de la democracia en nuestro país es esencial que los ciudadanos tomen conciencia de la relación entre sus prácticas cotidianas - por más inocuas que pudieran parecerles a primera vista, como es el copiar en un examen-y las prácticas de sus gobernantes. Sólo existirá una buena democracia si sus ciudadanos son capaces de ejercer su pensamiento crítico al evaluar las prácticas de todos los integrantes de dicha democracia, y en buscar discutir abierta y vehementemente, las acciones que disminuyen la integridad de un pueblo. Los niños, jóvenes y adultos que se comprometan a buscar con sincero esfuerzo la verdad y la justicia, sin temer juzgar y debatir sus creencias habituales, incluida la gravedad de la deshonestidad académica, podrán mejorar la calidad de su democracia.

\section{REFERENCIAS}

Balbuena, S. E. \& Lamela, R. A. (2015). Prevalence, Motives, and Views of Academic Dishonesty in Higher Education. Asia Pacific Journal of Multidisciplinary Research, 3(2), 69-75.

Bandura, A. (1999). Moral disengagement in the perpetration of inhumanities. Personality \& Social Psychology Review, 3(3), 193-209.

Basu, K. (2011). Why, for a Class of Bribes, the Act of Giving a Bribe should be Treated as Legal. MPRA Paper No. 50335. Disponible en http://mpra.ub.uni-muenchen.de/50335/

Buscaglia, E. \& van Dijk, J. (2003). Controlling Organized Crime and Corruption in the Public Sector. Forum on Crime and Society, 3(1\&2), 3-34.

Canache, D. \& Allison, M. E. (2005). Perceptions of Political Corruption in Latin American Democracies. Latin American Politics and Society, 47(3), 91-111.

Denisova-Schmidt, E., Huber, M. \& Leontyeva, E. (2016). On the development of students' attitudes towards corruption and cheating in Russian universities. European Journal of Higher Education, 6(2), 128-143.

Diez-Martínez, E. (2015). Deshonestidad académica de alumnos y profesores. Su contribución en la desvinculación moral y corrupción social. Sinéctica (44), 1 16. 
Drury, A.C., Krieckhaus, J. \& Lusztig, M. (2006). Corruption, Democracy, and Economic Growth. International Political Science Review, 27(2), 121-136.

Glaeser, E. L., La Porta, R., Lopez-de-Silanes, F. \& Shleifer, A. (2004). Do Institutions Cause Growth? Journal of Economic Growth, 9(3), 271-303.

Johnston, M. (2012). Building a Social Movement Against Corruption. Brown Journal of World Affairs, 18(11), 57-74.

Johnston, M. (2014). Corruption Contention and Reform: The Power of Deep Democratization. Cambridge: Cambridge University Press.

Lederman, D., Loayza, N. V. \& Soares, R. R. (2006). On the Political Nature of Corruption. En R. Stapenhurst, N. Johnston \& R. Pelizzo (eds.), The Role of Parliament in Curbing Corruption (pp. 27-40). Washington, D.C.: The World Bank.

Mialon, H. M. \& Mialon, S. H. (2013). Go Figure: The Strategy of Nonliteral Speech. American Economic Journal: Microeconomics, 5(2), 186-212.

Mill, J. S. (1999). Sobre las libertades. Madrid: Alianza editores.

Rose-Ackerman, S. \& Palifka, B. J. (2016). Corruption and Government: Causes, Consequences, and Reform (Second Edition). Cambridge: Cambridge University Press.

Sen, A. (2012). La idea de la justicia. Madrid: Taurus.

Taylor, C. (2018). Corruption and Anticorruption in Democratic Athens. En R. Kroeze, A. Vitória \& G. Geltner (eds.), Anticorruption in History: From Antiquity to the Modern Era (pp. 21-33). Oxford: Oxford University Press.

Treisman, D. (2000). The causes of corruption: a cross-national study. Journal of Public Economics, 76(3), 399-457.

Van Berkel, M. (2018). Fighting Corruption between Theory and Practice: The Land of the Euphrates and Tigris in Transition, Ninth to Eleventh Centuries. En R. Kroeze, A. Vitória \& G. Geltner (eds.), Anticorruption in History: From Antiquity to the Modern Era (pp. 65-76). Oxford: Oxford University Press.

Watts, J. (2018). The Problem of the Personal: Tackling Corruption in Later Medieval England, 1250-1550. En R. Kroeze, A. Vitória \& G. Geltner (eds.), Anticorruption in History: From Antiquity to the Modern Era (pp. 91-102). Oxford: Oxford University Press.

Welsh, D.T., Ordóñez, L. D., Snyder, D. G. \& Christian, M. S. (2015). The Slippery Slope: How Small Ethical Transgressions Pave the Way for Larger Future Transgressions. Journal of Applied Psychology, 100(1), 114-27.

Sumario: 1. La deshonestidad académica es el inicio de la futura desvinculación moral; 2. La deshonestidad académica y la democracia; 3. Actividades realizadas con estudiantes para determinar la relación entre la corrupción y la deshonestidad académica; 4. Evidencia estadística de la incongruencia respecto a la deshonestidad académica; Conclusiones; Referencias. 